\title{
Phase-noise Reduction Through an External High-Q Network Using a Black-Box Oscillator Model
}

\author{
M. Pontón, F. Ramírez, A. Herrera, A. Suárez \\ DICOM, University of Cantabria, Spain
}

\begin{abstract}
A method is presented to minimize the phase noise of an existing oscillator when connected to an external high-Q network. It relies on the experimental extraction of a reducedorder model of the original free-running oscillator, which involves a characterization of its pulling effects. With this model it is possible to predict the solution curves, stability properties and phase-noise of the augmented oscillator. It enables the optimization of the high-Q network to maximize the phase-noise reduction at a stable operation point in a single valued section of the solution curve. The method has been applied to (VCO) MiniCircuits ROS-3000-819+ (2-3GHz) obtaining a phase-noise reduction of about $10 \mathrm{~dB}$ with respect to the free-running values.

Keywords —oscillator, phase noise, dielectric resonator.
\end{abstract}

\section{INTRODUCTION}

Several works demonstrate the possibility to reduce the phase noise of an existing oscillator though the connection of a high quality-factor (Q) network to its output port or as an additional feedback loop [1]-[4]. The advantage of this procedure is that no modification of the original oscillator design is needed since the high-Q network is introduced externally. However, the parameters of the added network must be designed judiciously. Otherwise, it can give rise to unstable behaviour, as demonstrated in [1]-[2]. Under the high Q required to achieve a significant phase-noise reduction, the added network exhibits a high frequency sensitivity and, as a result, is susceptible to give rise to multivalued curves when varying a parameter. Thus, the behaviour of the augmented oscillator must be accurately predicted to achieve a significant phase-noise reduction in a stable single-valued section of the solution curve. The method in [4] enables an optimization of the network parameters using a formulation based on a reducedorder model of the oscillator circuit. The external network should not significantly perturb the amplitude and frequency of this original oscillator, so the model is constituted by the derivatives of the admittance function of the oscillator circuit, calculated at its original free-running operation point. In [4], this oscillator model is extracted from harmonic-balance (HB) simulations at circuit level, applying finite differences to an auxiliary generator. However, if the oscillator is a commercial one, it will not be possible to simulate it in HB. On the other hand, if the oscillator is home-made but the models of its components are not reliable, the model extracted from HB will not be accurate enough.

In [5], a method was presented for the empirical extraction of an oscillator model using an injection-locking procedure. However, this model does not contain all the parameters required to predict the behavior of the oscillator augmented with a high-Q network. Here a different modelling procedure, based on the characterization of the oscillator pulling, is presented. This model will be applied to predict the solution curves, stability and phase noise of a commercial oscillator loaded with a high-Q network. The external networks in [4] contain slow-wave structures. Here the investigation will be extended to a high-Q network containing a dielectric resonator. This will enable a theoretical analysis of the effect of the $\mathrm{Q}$ factor, as well as a validation of the solution curves versus the continuous variation of a network parameter. The network will be tested on a Mini-Circuits ROS-3000-819+ VCO (2-3GHz). A second network containing a slow-wave structure [4], easier to integrate, will also be considered. Variations in the VCO frequency will be carried out, which will require the calculation of the oscillator model at different bias voltages.

\section{AUGMENTED OSCILLATOR WITH AN EMPIRICAL MODEL}

Let us consider an existing oscillator terminated in the load $Y_{o}=1 / 50 \mathrm{Ohm}^{-1}$. This load will be replaced with a one-port high-Q network having the input admittance $Y_{1}(\omega)$ (Fig. 1). Then, the total admittance of the oscillator is [4]:

$$
Y_{T}(V, \omega)+\left[Y_{1}(\omega)-Y_{o}\right]=Y_{T}(V, \omega)+Y_{L}(\omega)=0
$$

where $Y_{T}(V, \omega)$ is the total admittance function of the original oscillator, fulfilling $Y_{T}\left(V_{o}, \omega_{o}\right)=0$ at the free-running amplitude $V_{o}$ and frequency $\omega_{o}$, and $Y_{L}(\omega)$ is the increment in the load admittance (with respect to $Y_{o}$ ), due to the introduction of the one-port network. This network should not significantly affect the original oscillation amplitude and frequency, so it will be possible to expand $Y_{T}(V, \omega)$ in (1) in a first-order Taylor series about $V_{o}, \omega_{o}$. Note that $Y_{L}(\omega)$ is not expanded since, due to the high $\mathrm{Q}$, a high frequency sensitivity is expected. Splitting (1) into real and imaginary parts and solving for $\omega-\omega_{o}$ and $V-V_{o}$ one obtains [4]:

$$
\begin{aligned}
& E(\omega)=\omega-\omega_{o}-\frac{\partial Y_{r}}{\partial V} \frac{Y_{L}^{i}(\omega)}{\operatorname{det}_{o}}+\frac{\partial Y_{i}}{\partial V} \frac{Y_{L}^{r}(\omega)}{\operatorname{det}_{o}}=0 \\
& V=V_{o}-\frac{\partial Y_{i}}{\partial \omega} \frac{Y_{L}^{r}(\omega)}{\operatorname{det}_{o}}+\frac{\partial Y_{r}}{\partial \omega} \frac{Y_{L}^{i}(\omega)}{\operatorname{det}_{o}}
\end{aligned}
$$

where the derivatives are calculated at the free-running solution of the original oscillator circuit, $r$ and $i$ indicate real and imaginary parts, $E(\omega)$ is an error function and $\operatorname{det}_{o}$ is: 


$$
\operatorname{det}_{o}=\frac{\partial Y_{r}}{\partial V} \frac{\partial Y_{i}}{\partial \omega}-\frac{\partial Y_{r}}{\partial \omega} \frac{\partial Y_{i}}{\partial V}
$$

To predict the solution curves, one will need a reducedorder model constituted by the following quantities:

$$
\text { Osc model }=\left\{\omega_{o}, V_{o}, \frac{\partial Y}{\partial V}, \frac{\partial Y}{\partial \omega}\right\}
$$

To solve (2) both the amplitude and phase of the two derivatives in (4) are required, unlike the situation in [5]. Once the numerical model is available, it will be introduced into (2) (a). Then, the solution curve can be easily obtained by (i) performing a linear simulation of the high-Q network (which can be an electromagnetic one) to calculate $Y_{L}(\omega)$ and (ii) tracing the zero-error contour $E(\omega)=0$.

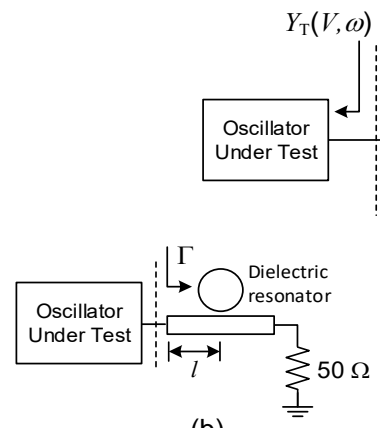

(b) (a)

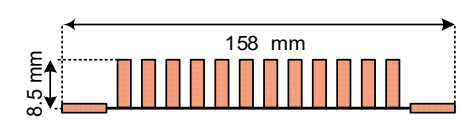

(c)
Fig. 1. Oscillator loaded with a high Q output network. (a) Use of a dielectric resonator $\left(\mathrm{r}=12 \mathrm{~mm}, \mathrm{~h}=12 \mathrm{~mm}, \varepsilon_{\mathrm{r}}=38\right)$ for the theoretical investigation. (b) Slow-wave structure.

The derivatives of the oscillator admittance function with respect to amplitude and frequency can be experimentally obtained by characterizing the pulling effects of the oscillator circuit. The oscillator is terminated with several loads having a small reflection coefficient and giving rise to the increments $Y_{L}(\omega)$. The resulting oscillation amplitudes and frequencies $\omega_{m}\left[Y_{L, m}\left(\omega_{m}\right)\right], V_{m}\left[Y_{L, m}\left(\omega_{m}\right)\right]$, where $m$ is a counter, are measured and stored. Using (2), one can solve for $(\partial Y / \partial V) / \operatorname{det}_{o}$ and $(\partial Y / \partial \omega) / \operatorname{det}_{o}$ from any two oscillation frequencies $\omega_{m}, \omega_{p}$ and output amplitudes $V_{m}, V_{p}$ : $\left[\begin{array}{l}\frac{\partial Y_{r}}{\partial V} \frac{1}{\operatorname{det}_{o}} \\ \frac{\partial Y_{i}}{\partial V} \frac{1}{\operatorname{det}_{o}} \\ \frac{\partial Y_{r}}{\partial \omega} \frac{1}{\operatorname{det}_{o}} \\ \frac{\partial Y_{i}}{\partial \omega} \frac{1}{\operatorname{det}_{o}}\end{array}\right]=\frac{1}{\Delta}\left[\begin{array}{cccc}Y_{L}^{r}\left(\omega_{p}\right) & -Y_{L}^{r}\left(\omega_{m}\right) & 0 & 0 \\ Y_{L}^{i}\left(\omega_{p}\right) & -Y_{L}^{i}\left(\omega_{m}\right) & 0 & 0 \\ 0 & 0 & -Y_{L}^{r}\left(\omega_{p}\right) & Y_{L}^{r}\left(\omega_{m}\right) \\ 0 & 0 & -Y_{L}^{i}\left(\omega_{p}\right) & Y_{L}^{i}\left(\omega_{m}\right)\end{array}\right]\left[\begin{array}{l}\omega_{m}-\omega_{o} \\ \omega_{p}-\omega_{o} \\ V_{m}-V_{o} \\ V_{p}-V_{o}\end{array}\right]$

$$
\Delta=Y_{L}^{i}\left(\omega_{m}\right) Y_{L}^{r}\left(\omega_{p}\right)-Y_{L}^{r}\left(\omega_{m}\right) Y_{L}^{i}\left(\omega_{p}\right)
$$

The above procedure is automated with a load-pull measurement [6]. A sufficiently large number of points enables an evaluation of the consistency of the experimental results. To calculate $\operatorname{det}_{o}$ one can injection-lock the oscillator circuit, introducing an independent locking signal at the output port, where the high-Q network will be connected. For a small injection-current amplitude $I_{g}$, the oscillator can be linearized about its steady-state solution [5]. The magnitude of the frequency derivative $|\partial Y / \partial \omega|$ can be obtained from the locking bandwidth $\Delta \omega_{\max }$ as:

$$
\left|\frac{\partial Y}{\partial \omega}\right|=\frac{2 I_{g}}{V_{o} \Delta \omega_{\max } \sin \alpha_{v \omega}}
$$

where $\alpha_{v \omega}$ is the difference between the angle of $\partial Y / \partial \omega$ and that of $\partial Y / \partial V$, easily obtained from (5). Then, the quantity det $_{0}$, which should be a positive scalar in a well behaved oscillator, is directly calculated combining (2) and (6). The procedure allows obtaining an empirical model of the standalone oscillator, shown in (4), which will be used to calculate the steady-state solution of the augmented oscillator [from (2)], as well as its stability and phase noise. The method has been applied to the (VCO) Mini-Circuits ROS-3000-819+ $(2-3 \mathrm{GHz})$. This particular oscillator is very convenient because, as reported in [7], it is unbuffered. Table I shows the model obtained when biasing the $\mathrm{VCO}$ at $V_{t}=2.45 \mathrm{~V}$, which provides the free-running frequency $f_{o}=2.151 \mathrm{GHz}$.

\begin{tabular}{|c|c|}
\multicolumn{2}{|c|}{ Table I. Oscillator model } \\
\hline Parameter & Value \\
\hline $\mathrm{V}_{0}$ & $0.6 \mathrm{~V}$ \\
\hline $\mathrm{f}_{0}$ & $2.15 \mathrm{GHz}$ \\
\hline$\partial Y / \partial \omega$ & $7.69 \mathrm{E}-11+3.82 \mathrm{E}-10 \cdot \mathrm{i}$ \\
\hline$\partial Y / \partial V$ & $0.1538-0.1995 \mathrm{i}$ \\
\hline
\end{tabular}

In the following, two different types of output network will be considered. The first one, containing a dielectric resonator coupled to a transmission line [Fig. 1(b)], will allow a simple experimental validation of the solution curves obtained when varying a network parameter in a continuous manner. Even though, in practice, other configurations may be more convenient, this one will allow getting analytical insight into the whole circuit behaviour. The second one [Fig. 1(c)] is based on a slow-wave structure, as in [4], where a home-made oscillator circuit and a model extracted from HB simulations are used.

\section{STRUCTURE WITH A DIELECTRIC RESONATOR}

Initially, an analytical derivation of the oscillation frequency in the presence of a high-Q resonator will be carried out. For simplicity of this analytical derivation, the structure is made up of just dielectric resonator coupled to a transmission line [8]-[9]. From [8] the input reflection coefficient is:

$$
\Gamma=\frac{k \omega_{o}}{(k+1) \omega_{o}+2 j Q\left(\omega-\omega_{o}\right)} e^{-2 j \frac{\omega l}{v}}
$$

where $k$ is the coupling factor, $\mathrm{Q}$ is the quality factor, $\omega_{o}$ is the resonance frequency and $l$ is the length of the transmission line from the input reference plane to the coupling point [8]. Using (7) to calculate $Y_{L}$ and replacing the resulting expression into (2) (a), the oscillation frequency can be calculated from: 


$$
\begin{aligned}
& \alpha\left(\omega-\omega_{o}\right)+4 Q^{2}\left(\omega-\omega_{o}\right)^{3}+ \\
& \frac{2 Y_{o}}{\operatorname{det}_{o}}\left(\begin{array}{l}
{\left[\frac{\partial Y_{r}}{\partial V} \gamma-\frac{\partial Y_{i}}{\partial V} \xi Q\left(\omega-\omega_{o}\right)\right] \sin \left(\frac{2 \omega l}{v}\right)} \\
+\left[\frac{\partial Y_{i}}{\partial V} \gamma-\frac{\partial Y_{r}}{\partial V} \xi Q\left(\omega-\omega_{o}\right)\right] \cos \left(\frac{2 \omega l}{v}\right)
\end{array}\right)=0
\end{aligned}
$$

where $\gamma=(k+1) k \omega_{o}^{2}, \xi=2 k \omega_{0}, \alpha=(k+1)^{2} \omega_{o}^{2}, v=c / \sqrt{\varepsilon_{\text {eff }}}$ As gathered from (8), when increasing $\mathrm{Q}$, multivalued solutions in terms of the oscillation frequency $\omega$ versus the length $l$ can arise, and the frequency excursion decreases. The frequency $\omega$ agrees with the free-running one at the $l$ values $\left(l_{p}\right)$ fulfilling:

$$
\tan \left(2 \omega_{o} l_{p} / v\right)=-\frac{\partial Y_{i}}{\partial V} / \frac{\partial Y_{r}}{\partial V}
$$

Fig. 2 presents the variation of the oscillation frequency versus the length $l$. The results of the approximate model are compared with those obtained with the numerical technique in (2)(a), based on a linear simulation of the output network (providing $Y_{L}$ ) with very good agreement. Fulfilment of (9) can be noted. Length values $l_{p}$ in single- and multi-valued regions alternate Experimental measurements are also superimposed in Fig. 2. Note that in the theoretical curves $Y_{L}$ is calculated either from (7) or through a linear simulation. It is not empirical, which justifies the small discrepancies.

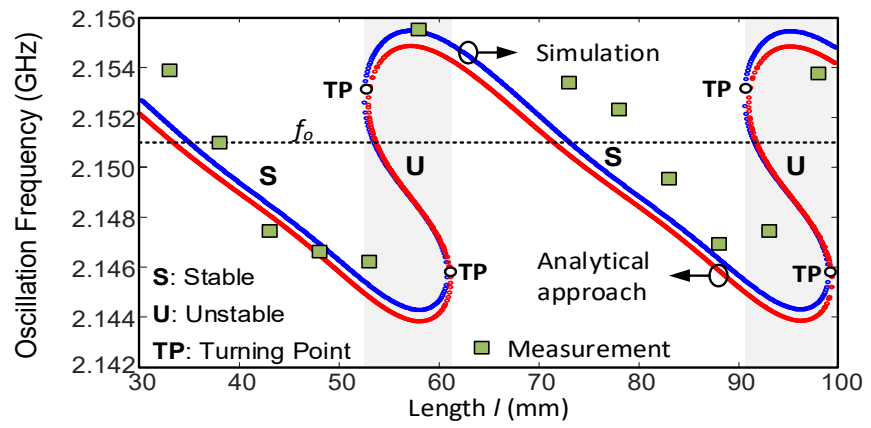

Fig. 2. Solution curve of the Mini-Circuits ROS-3000-819+ VCO $(2-3 \mathrm{GHz})$ when loaded with a transmission line coupled to a high $\mathrm{Q}$ resonator. The curve has been obtained using the empirical model. Measurement points are superimposed.

A detailed characterization of the first predicted discontinuous jump, with a much finer variation of the length $l$, is presented in Fig. 3. The measurement has been performed by holding the spectral-line peak, while sliding the dielectric resonator along the transmission line.

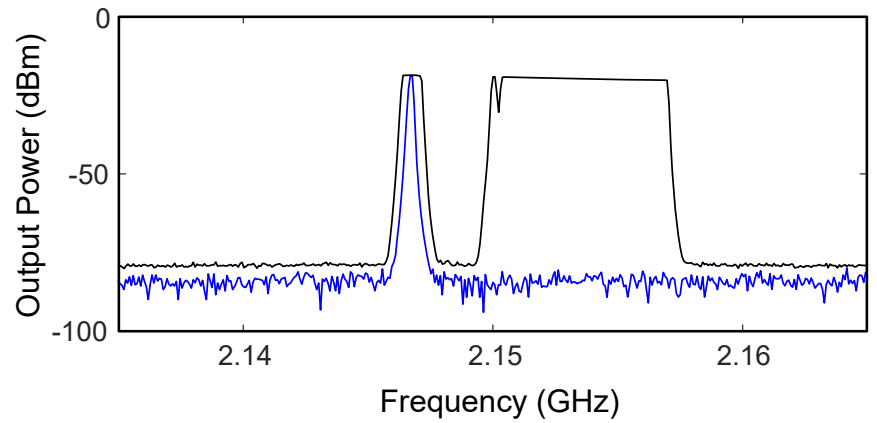

Fig. 3. Detailed characterization of the discontinuous jump predicted in Fig. 2.
A stability analysis has also been carried out, using the method in [4], combined with the new experimental model of the standalone oscillator. The solution is stable, except in its multi-valued sections, where there is a coexistence of stable and unstable solutions due to the presence of turning points (TP) (Fig. 2). The variation of the solution poles versus the length $l$ is shown in Fig. 4(a). The phase-noise spectral density is given by [4]:

$$
\left\langle|\delta \phi|^{2}\right\rangle=\frac{1}{\Omega^{2}} \frac{\left|\frac{\partial Y}{\partial V}\right|^{2} \frac{2\left|I_{w}\right|^{2}+k / \Omega}{V_{o}^{2}}}{\left(\operatorname{det}_{o}+\frac{\partial Y_{r}}{\partial V} \frac{\partial Y_{L}^{i}}{\partial \omega}-\frac{\partial Y_{i}}{\partial V} \frac{\partial Y_{L}^{r}}{\partial \omega}\right)^{2}}
$$

where $\Omega$ is the offset frequency from the carrier. The equivalent noise source $2\left|I_{w}\right|^{2}+k / \Omega$ (including the upconverted flicker component) is derived by fitting the power spectral density of the free-running oscillator. As gathered from (7) and (8), when varying the length $l$, the frequency derivative of $Y_{L}$ will exhibit an oscillatory behavior, so one can expect to obtain phase-noise minima and maxima. A high phase-noise reduction will be due to the high magnitude exhibited by the term added to det $_{0}$ in the denominator of (10) (for some $l$ values) under a high $\mathrm{Q}$, as easily derived from (7) and (8). Fig. 4(b) presents the variation of the phase-noise spectral density at $10 \mathrm{kHz}$. Measurement points are superimposed. The experimentally characterized phase-noise spectrum, in free-running conditions and with the resonator at $l=83 \mathrm{~mm}$, is shown in Fig. 5, where it is compared with the one obtained in simulation. As shown in Fig. 4(b) and Fig. 5, a significant phase-noise improvement is achieved.

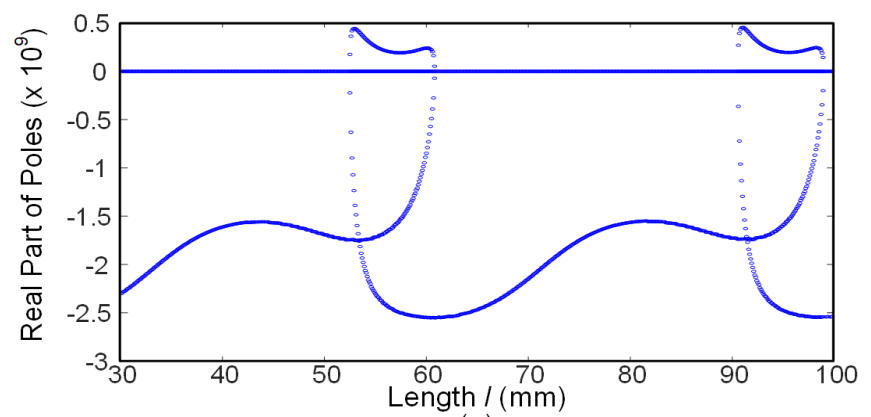

(a)

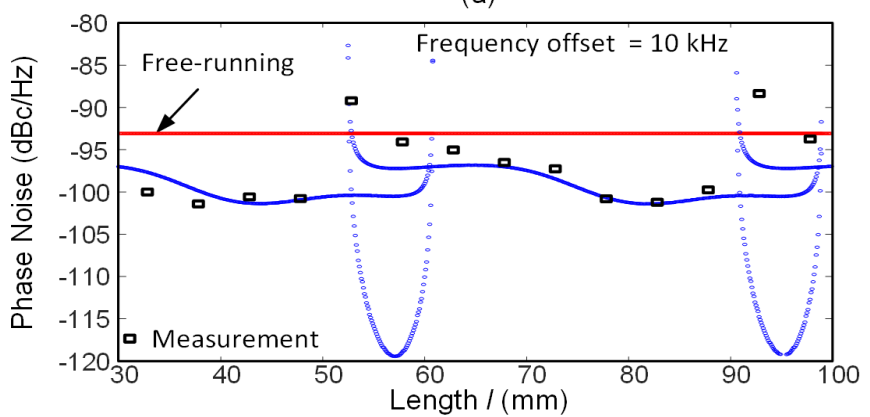

(b)

Fig. 4. Stability and noise properties, using the empirical model. (a) Stability analysis versus $l$. (b) Variation of the phase-noise spectral density at $10 \mathrm{kHz}$. 


\section{PHASE-NOISE REDUCTION WITH SLOW-WAVE STRUCTURE}

In a second experiment, the oscillator has been loaded with a one-port network containing a slow-wave structure in a reflection configuration [4]. The structure is based on a simple distributed implementation of the inductors and capacitors in a discrete transmission line [Fig. 1(c)] and provides a group delay of $3.1 \mathrm{~ns}$ at $2.45 \mathrm{GHz}$. Unlike the case in Section III (when using the dielectric resonator) it is not possible to vary any parameters of the slow-wave structure. Thus, the external network must be properly optimized to enable the phase-noise reduction at the desired oscillation frequency. Otherwise, the phase noise can even increase. Variations in the VCO bias voltage between $V_{t}=2 \mathrm{~V}$ and $9 \mathrm{~V}$ are considered, which requires obtaining the standalone-oscillator model at different $V_{t}$ values: $\left\{\omega_{o}\left(V_{t}\right), V_{o}\left(V_{t}\right), Y_{V}\left(V_{t}\right), Y_{\omega}\left(V_{t}\right)\right\}$, where the subscripts $V$ and $\omega$ indicate the variable with respect to which the derivative is calculated. The noise source in (10) is fitted for each $V_{t}$.

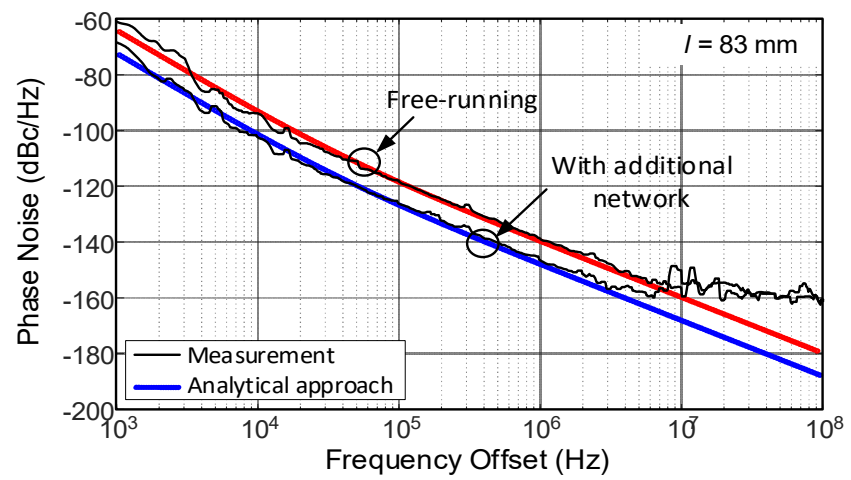

Fig. 5. Phase-noise spectrum at $l=83 \mathrm{~mm}$. The spectrum is compared with the one obtained in free-running conditions.

Fig. 6(a) presents the phase-noise spectral density versus the bias voltage $V_{t}$ at the constant offset frequency $100 \mathrm{kHz}$. Note that simulations were only carried out at the discrete $V_{t}$ values for which the experimental oscillator model was obtained. The results are compared with a continuous measurement of the phase-noise spectral density at the same offset frequency. As expected, there are minima and maxima. The phase-noise measurements in free-running conditions are also presented. The spectral density measured at $V_{t}=5.55 \mathrm{~V}$ with and without the slow-wave structure is shown in Fig. 6(b). The resulting values are $-118.6 \mathrm{dBc} / \mathrm{Hz}$ and $-127.8 \mathrm{dBc} / \mathrm{Hz}$, at $100 \mathrm{kHz}$, respectively, and $-139.2 \mathrm{dBc} / \mathrm{Hz}$ and $-148.65 \mathrm{dBc} / \mathrm{Hz}$, at $1 \mathrm{MHz}$.

\section{CONCLUSIONS}

A method has been proposed to predict the phase-noise reduction of an existing oscillator, modeled as a black box, when connected to a high Q output network. The model is easily obtained from a characterization of the oscillator pulling effects. The capability to predict the solution curves versus a parameter of the external network has been tested using a dielectric resonator coupled to a transmission line. The relative simplicity of this load network has allowed an analytical derivation of the solution curves, which become multivalued for some parameter intervals. Then, a slow-wave structure has been used, considering variations in the oscillator bias voltage. Phase-noise reductions of about $10 \mathrm{~dB}$ have been achieved.

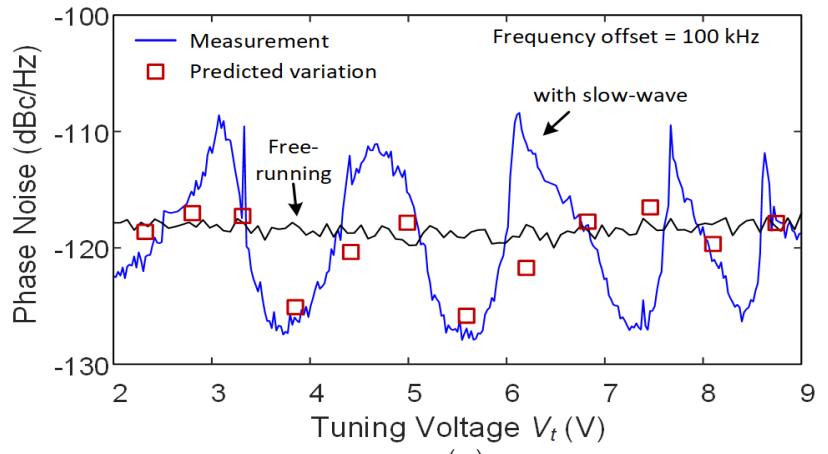

(a)

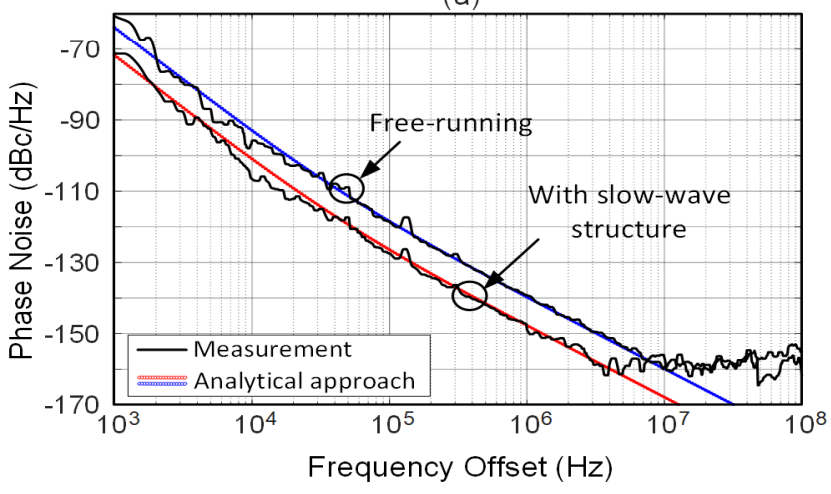

(b)

Fig. 6. Phase noise spectral density when loading the oscillator with a network containing a slow-wave structure. (a) Variation of the spectral density at 100 $\mathrm{kHz}$ versus $V_{t}$ with and without the slow-wave structure. (b) Measured phasenoise spectrum at $V_{t}=5.55 \mathrm{~V}$.

\section{ACKNOWLEDGMENT}

Work supported by the Spanish Ministry of Science and Innovation (ERDF/FEDER) project TEC2017-88242-C3-1-R.

\section{REFERENCES}

[1] H.-C. Chang, "Phase noise in self-injection-locked oscillators - theory and experiment," IEEE Trans. Microw. Theory Techn., vol. 51, no. 9, pp. 1994-1999, Sept. 2003.

[2] A. Suárez, F. Ramírez, "Analysis of Stabilization Circuits for PhaseNoise Reduction in Microwave Oscillators," IEEE Trans. Microw. Theory Techn., vol. 53, no. 9, pp. $2743-2751$, Sep. 2005.

[3] K. Peng, C. Lee, D. Wong, F. Wang and T. Horng, "An Injection- and Frequency-Locked Loop for Reducing Phase Noise of Wideband Oscillators," IEEE Trans. Microw. Theory Techn, vol. 66, no. 3, pp. 1374-1383, March 2018.

[4] M. Pontón, F. Ramírez, A. Herrera, A. Suárez, "Oscillator Stabilization through Feedback With Slow Wave Structures," IEEE Trans. Microw. Theory Techn., vol. 67, 2020.

[5] F. Ramírez, S. Sancho, M. Pontón, A. Suárez, "Two-Scale EnvelopeDomain Analysis of Injected Chirped Oscillators," IEEE Trans. Microw. Theory Techn., vol. 66, no. 12, pp. 5449-5461, Dec. 2018.

[6] C.Tsironis, "Oscillator Load Pull Measurements Using a Computer Controlled Tuner,” 35ARFTG Conf., Dallas, TX, USA, 1990, pp. 12-18.

[7] Buchanan, N.B., Fusco, V., "Single VCO chipless RFID near-field reader," Electron. Lett., vol. 52, no. 23, pp. 1958-1960, 2016.

[8] R. Soares, J. Graffeuil , J. Obregon, "Applications des transistors à effet de champ en arséniure de gallium," Collection Technique et Scientifique des Télécommunications, Eyrolles, 1984.

[9] T. Makino, A. Hashima, "A Highly Stabilized MIC Gunn Oscillator Using a Dielectric Resonator," IEEE Trans. Microw. Theory Techn., vol. 27, no. 7, pp. 633-638, Jul., 1979. 